\title{
Theory of Discrete Time SISO Linear $(L, M)$ Shift Invariant System
}

\author{
Wing-kuen Ling and P. K. S. Tam
}

\author{
Department of Electronic and Information Engineering \\ The Hong Kong Polytechnic University \\ Hung Hom, Kowloon, Hong Kong \\ Hong Kong Special Administrative Region, China \\ Tel: (852) 2766-6238, Fax: (852) 2362-8439 \\ Email: bingo@encserver.eie.polyu.edu.hk, enptam@polyu.edu.hk
}

\begin{abstract}
In this paper, we have characterized the discrete time single input single output (SISO) linear $(L, M)$ shift invariant system by a two-dimensional kernel function and a filter bank structure. Based on the characterization, we have investigated the conditions for the stability, the invertibility, the causality and the finite response properties of a discrete time SISO linear $(L, M)$ shift invariant system. The advantages of the analysis is that a linear time varying system can be analyzed and designed through a finite number of one-dimensional kernel functions and linear time invariant (LTI) filters. Hence, it facilitates the analysis and the design of a linear time varying system, such as an $L / M$ rate changer used in the digital image processing and digital video processing.
\end{abstract}

Index Terms-discrete time SISO linear $(L, M)$ shift invariant system, kernel function, filter bank, time varying system, $L / M$ rate changer

\section{INTRODUCTION}

A lot of discrete time SISO systems are LTI, such as, those systems governed by constant coefficients difference equation. Those systems are completely characterized by the impulse response and the frequency response. Besides the discrete time SISO LTI systems, some discrete time SISO systems are linear time periodic varying (LTPV) [1, 2], such as the sequential switching systems. Those systems play an important role in the research in this decade.

However, it is found that there are some discrete time SISO linear systems, which the output will shift by $L$ samples if the input shifts by $M$ samples ( $L$ and $M$ are the minimum positive integers), those systems are known as discrete time SISO linear $(L, M)$ shift invariant systems. It is found that those systems are a more general class of discrete time SISO linear time varying systems including the discrete time SISO LTI systems and discrete time SISO LTPV systems. Those systems cannot be characterized by the impulse response and the frequency response. Hence, the stability, the invertibility, the causality and the finite response properties are not determined.

In this paper, we have characterized all the discrete time SISO linear $(L, M)$ shift invariant system and figure out the conditions for the stability, the invertibility, the causality and the finite response properties.

\section{RELATIONSHIP AMONG DISCRETE TIME SISO LTI SYSTEM, DISCRETE TIME SISO LTPV SYSTEM AND DISCRETE TIME SISO LINEAR $(L, M)$ SHIFT INVARIANT SYSTEM}

For a discrete time SISO LTI system, the output will shift by one sample if the input shifts by one sample also. Hence, a discrete time SISO LTI system is a discrete time SISO linear $(L, M)$ shift invariant system with $L=M=1$. For a discrete time SISO LTPV system with period $M$, the output will shift by $M$ samples if the input shifts by $M$ samples where $M$ is the period of the system. Hence, a discrete time SISO LTPV system is also a discrete time SISO linear $(L, M)$ shift invariant system with $L=M$. In general, the discrete time SISO linear $(L, M)$ shift invariant system is a more general class of discrete time SISO time varying system including the discrete time SISO LTI system and discrete time SISO LTPV system. The relationship among these three kinds of systems is summarized in figure 1.

\section{CHARACTERIZATION OF DISCRETE TIME SISO LINEAR $(L, M)$ SHIFT INVARIANT SYSTEM}

A discrete time SISO system is LTI if and only if the output is the convolution sum of the impulse response and the input. Hence, the impulse response can completely characterize a discrete time SISO LTI system. However, the impulse response cannot be used to characterize the discrete time SISO LTPV system and the discrete time SISO linear $(L, M)$ shift invariant system. In fact, it can be characterized by the following two theorems:

Theorem 1

A system is discrete time SISO linear $(L, M)$ shift invariant system if and only if there exists a two-dimensional kernel function $g[n, k]$ such that the input output relationship of the system is governed by:

$$
y[n]=\sum_{k \rightarrow-\infty}^{+\infty} g[n, k] \cdot x[k] \text { where } g[n, k]=g[n-L, k-M]
$$




\section{Theorem 2}

A system is discrete time SISO linear $(L, M)$ shift invariant system if and only if there exists some discrete time SISO LTI filters, $h_{j}[k]$, for $j=0,1, \ldots, L-1$, such that the discrete time SISO linear $(L, M)$ shift invariant system can be realized as in figure 2.

The details proof is not intended to include in this paper.

\section{PROPERTIES OF DISCRETE TIME SISO LINEAR $(L, M)$ SHIFT INVARIANT SYSTEM}

As a discrete time SISO linear $(L, M)$ shift invariant system can be characterized by the two-dimensional kernel function $g[n, k]$ and the filter bank system shown in figure 2 . Hence, the stability, the invertibility, the causality and the finite response properties of a discrete time SISO linear $(L, M)$ shift invariant system can be figured out and summarized as the following theorems:

\section{Theorem 3}

A discrete time SISO linear $(L, M)$ shift invariant system is bounded input bounded output (BIBO) stable if and only if the corresponding filters in the filter bank system shown in figure 2 , that is, $h_{j}[k]$, for $j=0,1, \ldots, L-1$, are all stable.

\section{Theorem 4}

A linear $(L, M)$ shift invariant system is BIBO stable if and only if $g\left[n_{0}, k\right], g\left[n_{1}, k\right], \ldots, g\left[n_{L-1}, k\right]$ are stable, where $\left\{\bmod \left(n_{0}, L\right), \bmod \left(n_{1}, L\right), \ldots, \bmod \left(n_{L-1}, L\right)\right\}=\{0,1, \ldots, L-1\} \quad$ and $\bmod (x, L)$ is defined as the reminder of $x / L$.

\section{Theorem 5}

A linear $(L, M)$ shift invariant system is invertible if there exists a set of discrete time SISO LTI filters $\left\{f_{0}[n]\right.$, $\left.f_{I}[n], \ldots, f_{L-1}[n]\right\}$ such that:

$\left[\begin{array}{cccc}H_{0}(z) & H_{1}(z) & \cdots & H_{L-1}(z) \\ H_{0}(z \cdot W) & H_{1}(z \cdot W) & \cdots & H_{L-1}(z \cdot W) \\ \vdots & \vdots & \ddots & \vdots \\ H_{0}\left(z \cdot W^{M-1}\right) & H_{1}\left(z \cdot W^{M-1}\right) & \cdots & H_{L-1}\left(z \cdot W^{M-1}\right)\end{array}\right] \cdot\left[\begin{array}{c}F_{0}(z) \\ F_{1}(z) \\ \vdots \\ F_{L-1}(z)\end{array}\right]=\left[\begin{array}{c}M \\ 0 \\ \vdots \\ 0\end{array}\right](2)$, where $W=e^{-\frac{j \cdot 2 \cdot \pi}{M}}$.

\section{Theorem 6}

A linear $(L, M)$ shift invariant system is invertible if there exists a set of discrete time SISO LTI filters $\left\{f_{0}[n]\right.$, $\left.f_{I}[n], \ldots, f_{L-1}[n]\right\}$ such that:

$\left[\begin{array}{cccc}G\left(0, \frac{1}{z}\right) & G\left(1, \frac{1}{z}\right) & \cdots & G\left(L-1, \frac{1}{z}\right) \\ G\left(0, \frac{1}{z \cdot W}\right) & G\left(1, \frac{1}{z \cdot W}\right) & \cdots & G\left(L-1, \frac{1}{z \cdot W}\right) \\ \vdots & \vdots & \ddots & \vdots \\ G\left(0, \frac{1}{z \cdot W^{M-1}}\right) & G\left(1, \frac{1}{z \cdot W^{M-1}}\right) & \cdots & G\left(L-1, \frac{1}{z \cdot W^{M-1}}\right)\end{array}\right] \cdot\left[\begin{array}{c}F_{0}(z) \\ F_{1}(z) \\ \vdots \\ F_{L-1}(z)\end{array}\right]=\left[\begin{array}{c}M \\ 0 \\ \vdots \\ 0\end{array}\right](3)$

\section{Theorem 7}

A linear $(L, M)$ shift invariant system is causal if and only if the corresponding filters in the filter bank system shown in figure 2 , that is, $h_{j}[k]$, for $j=0,1, \ldots, L-1$, are all causal.

\section{Theorem 8}

A linear $(L, M)$ shift invariant system is causal if and only if $g[n, k]=0$ for $k \cdot L>n \cdot M$.

\section{Theorem 9}

A linear $(L, M)$ shift invariant system has finite response if and only if the corresponding filters in the filter bank system shown in figure 2 , that is, $h_{j}[k]$, for $j=0,1, \ldots, L-1$, are all FIR filters.

\section{Theorem 10}

A linear $(L, M)$ shift invariant system has finite response if and only if $g\left[n_{0}, k\right], g\left[n_{1}, k\right], \ldots, g\left[n_{L-1}, k\right]$ are all FIR filters, where $\left\{\bmod \left(n_{0}, L\right), \bmod \left(n_{1}, L\right), \ldots, \bmod \left(n_{L-1}, L\right)\right\}=\{0,1, \ldots, L-1\}$.

The details proof is also not intended to include in this paper.

\section{CONCLUSION}

In this paper, a discrete time SISO linear $(L, M)$ shift invariant system is investigated. This kind of system includes a discrete time SISO LTPV system and a discrete time SISO LTI system. This is a wide class of system and has many applications, such as applied as a rate changer used in the multimedia systems. It is found that this kind of system can be characterized by a two-dimensional kernel function $g[n, k]$ or by a filter bank system shown in figure 2 . Based on the characterization, the stability, the invertibility, the causality and the finite response of a discrete time SISO linear $(L, M)$ shift invariant system can be determined in terms of this two-dimensional kernel function $g[n, k]$ or by the corresponding filters in the filter bank system shown in figure 2 , that is, $h_{j}[k]$, for $j=0,1, \ldots, L-1$. The advantages of this analysis is that such a discrete time SISO time varying system can be analyzed and designed by a finite number of discrete time SISO LTI filters.

\section{ACKNOWLEDGEMENT}

The work described in this letter was substantially supported by a grant from the Hong Kong Polytechnic University with account number G-V968.

\section{REFERENCES}

[1] Iraj Sodagar, Kambiz Nayebi, Thomas P. Barnwell, III, and Mark J. T. Smith, "Time-Varying Analysis-Synthesis Systems Based on Filter Banks and Post Filtering," IEEE Transactions on Signal Processing, vol. 43, No. 11, pp. 2512-2524, November, 1995.

[2] Iraj Sodagar, Kambiz Nayebi and Thomas P. Barnwell, III, "A Class of Time Varying Wavelet Transforms," IEEE International Conference on Acoustics, Speech, and Signal Processing, ICASSP, vol. 3, pp. 201-204, April, 1993. 


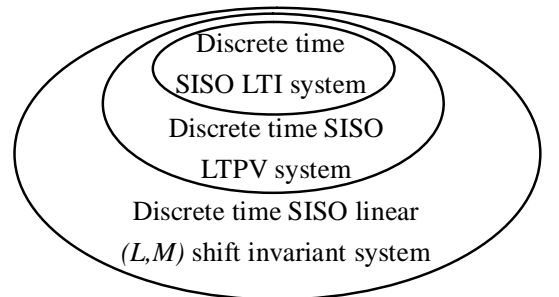

Fig. 1. Relationship among discrete time SISO LTI system, discrete time SISO LTPV system and discrete time SISO linear $(L, M)$ shift invariant system.

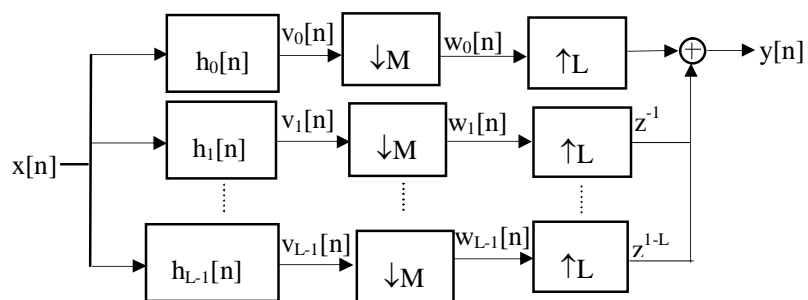

Fig. 2. Filter bank realization of discrete time SISO linear $(L, M)$ shift invariant system. 